\title{
Cefepime versus Ceftriaxone for Empiric Treatment of Hospitalized Patients with Community-Acquired Pneumonia
}

We read with interest the report of the clinical trial by Zervos et al. (5) in which cefepime was compared with ceftriaxone for empiric treatment of hospitalized patients with community-acquired pneumonia. Although that paper presented some interesting findings, there are several methodological flaws with the study that we would like to comment on.

The first problem relates to patient eligibility. The criteria for hospitalization of patients $(3,4)$ were not clearly defined. It appears that patients were included in the trial without radiological evidence of pneumonia, even though it has been suggested that a chest film showing infiltrates is necessary to establish the diagnosis of pneumonia (1). Moreover, the authors apparently also considered as a criterion for patient eligibility the presence of bacteria susceptible to cefepime and ceftriaxone. This seems rather risky since it has been shown that it is seldom possible to reach a specific etiologic diagnosis by roentgenography alone (4). We also found that in Table 2, which describes the characteristics of pneumonia presented by the patients at the time of entry in the study, the severity of pneumonia is classified as mild, moderate, or severe, but definitions for these categories are not provided.

A second difficulty encountered by the reader is that, in evaluating efficacy, the authors define the category "improvement" as "improvement of one or more signs and symptoms of pneumonia without a complete resolution but a lack of progression of radiographic findings," and consider it a favorable clinical response. This would mean that if a patient improved in just one symptom, say for example, cough, this would be regarded as a "favorable clinical response." We feel that the category "improvement" makes little medical sense in dealing with pneumonia. In addition, in the General Guidelines for the Evaluation of New Anti-Infective Drugs for the Treatment of Respiratory Tract Infections (2), this category is not included in the definition of clinical response. Moreover, it is not clear how the category "improvement" was determined for patients which were included in the study without a previous chest film.

Thirdly, we are also concerned about the fact that in this study some patients were treated concomitantly with several other antibiotics. Although treatment with erythromycin and metronidazole can be justified in cases where atypical pathogens or anaerobes are suspected, there were seven patients who received six additional antibiotics. Despite the fact that the authors suggest that antibiotic treatment had little impact on the efficacy of the drugs studied, it is difficult to classify these patients with respect to clinical response. Besides, the authors never mention when these additional antibiotics where added or what the criterion was for adding them. We feel that the criteria for patient evaluation in this study are debatable. For example, what exactly do the authors mean when they say that "Seven additional patients who received additional antimicrobial agents were approved by the sponsor as evaluable"?

Finally, this study also lacks a clearly stated hypothesis and, thus, a sample size calculation.

\section{REFERENCES}

1. Barlett, J. G., and L. M. Mundy. 1995. Community-acquired pneumonia. N. Engl. J. Med. 333:1618-1624.
2. Chow, A. W., C. B. Hall, J. O. Klein, R. B. Kammer, R. D. Meyer, and J. S. Remington. 1992. General guidelines for the evaluation of new anti-infective drugs for the treatment of respiratory tract infections. Clin. Infect. Dis. 15 (Suppl. 1):S62-S88.

3. Levison, M. E. 1998. Pneumonia, including necrotizing pulmonary infections (lung abscess), p. 1437-1445. In Harrison's principles of internal medicine, 14th ed. McGraw-Hill, New York, N.Y.

4. Penn, R. L., and R. F. Betts. 1996. Lower respiratory tract infections, p. 258349. In R. E. Reese and R. F. Betts (ed.), A practical approach to infectious diseases, 4th ed. Little, Brown and Company, Toronto, Canada.

5. Zervos, M., M. Nelson, and The Cefepime Study Group. 1998. Cefepime versus ceftriaxone for empiric treatment of hospitalized patients with community-acquired pneumonia. Antimicrob. Agents Chemother. 42:729-733.

\author{
Javier Borja \\ Francesc Jané* \\ Department of Clinical Pharmacology \\ Hospital de la Santa Creu i Sant Pau \\ Avgda. Sant Antoni $M^{a}$ Claret, 167 \\ 08025 Barcelona \\ Spain \\ *Phone: 34932919018 \\ Fax: 34932919178 \\ E-mail: 1273@hsp.santpau.es
}

\section{Author's Reply}

We disagree with Borja and Jané regarding the alleged methodological flaws in our study.

Regarding eligibility, previously published definitions of pneumonia $(1,2)$ were used. Evidence of community-acquired pneumonia consisted of documentation of at least two of the following three groups of signs and symptoms (i) fever (temperature greater than $38^{\circ} \mathrm{C}$ ) or hypothermia (temperature less than $\left.36^{\circ} \mathrm{C}\right)$; (ii) purulent sputum $(>25$ polymorphonuclear leukocytes and $<10$ squamous cells/low-power field), leukocytosis $\left(>10,000\right.$ leukocytes $/ \mathrm{mm}^{3}$ ), worsening arterial oxygen gradients in relation to baseline measurements, or newly developed hypoxia; and (iii) evidence of pulmonary involvement probably due to the presence of bacteria susceptible to cefepime and ceftriaxone (radiologic findings consistent with the diagnosis of aspiration pneumonia, lobar pneumonia, or diffuse unilateral or bilateral pneumonia and/or signs of pulmonary consolidation or pneumonia on physical examination). All patients had pulmonary infiltrates (Table 2 in our article). We agree that definitions used for severity of illness should have been included.

Regarding improvement not being a useful category, patients were considered to have improved if there was a lack of progression in all positive findings on chest radiography but improvement in one or more signs and symptoms of pneumonia without a complete resolution.

Regarding concomitant antibiotics, as stated in our article, erythromycin and metronidazole were administered for atypical pathogens and anaerobes, respectively. Patients that received concomitant antibiotics were analyzed separately. Although certain concomitant antibiotics (primarily erythromycin and metronidazole) were allowed during this trial, concomitant treatment appeared to have little impact on the rate of favorable clinical response in the clinically evaluated patients (cefepime group, 100\% with concomitant antibiotics and 
92.9\% without; ceftriaxone group, $92.9 \%$ with concomitant antibiotics and $100 \%$ without).

Regarding sample size determination, we acknowledged in Discussion that this trial did not include sufficient numbers of patients to detect subtle differences in efficacy and safety between cefepime and ceftriaxone; the data reported, however, support the conclusion that cefepime and ceftriaxone are therapeutically comparable for the empiric treatment of community-acquired pneumonia in hospitalized patients. These two agents were equally effective against the pathogens in this study, and both were associated with a low rate of adverse effects.

Finally, we feel the objective of this study was clear and obvious; it was, as stated, to evaluate the comparative safety and efficacy of cefepime and ceftriaxone for community-acquired pneumonia.

\section{REFERENCES}

1. Bartlett, J. G., R. F. Breiman, L. A. Mandell, and T. M. File, Jr. 1998. Community-acquired pneumonia in adults: guidelines for management. Clin. Infect. Dis. 26:811-838.

2. Chow, A. W., C. B. Hall, J. O. Klein, R. B. Kammer, R. D. Meyer, and J. S. Remington. 1992. General guidelines for the evaluation of new anti-infective drugs for the treatment of respiratory tract infections. Clin. Infect. Dis. 15 (Suppl. 1):S62-S88.

Marcus J. Zervos

William Beaumont Hospital Royal Oak, Mich. 